\title{
VERIFICAÇÃO DA PRÓTESE AUDITIVA REALIZADA FACE A FACE E VIA TELECONSULTA: MEDIDAS REPETIDAS
}

\author{
Telecounselling and face to face hearing aid verification: \\ repeated measures
}

\author{
Deborah Viviane Ferrari (1), Gabriela Rosito Alvarez Bernardez-Braga (2), Patrícia Danieli Campos ${ }^{(3)}$
}

\begin{abstract}
RESUMO
Objetivo: avaliar as medidas repetidas da resposta de ressonância da orelha externa sem (REUR) e com uso de amplificação (REAR) e o ganho de inserção (REIG), realizados face a face e via teleconsulta. Método: estudo prospectivo longitudinal. Foram realizadas quatro repetições da REUR, REAR e REIG (com estímulo speech noise apresentado em $65 \mathrm{~dB}$ NPS e $0^{\circ}$ azimute) em 19 orelhas de adultos ouvintes normais, via face a face $(F)$ e teleconsulta síncrona por controle remoto de aplicativo (T) e vídeo interativo. O software Polycom PVX foi utilizado para compartilhamento e transmissão de áudio e vídeo. A conexão foi realizada via LAN (Local Area Network) USP na velocidade de 384 kbps Foi calculado o erro causal (Dalhberg) entre as quatro medidas para frequências de 250 a 8000 $\mathrm{Hz}$. Resultados: os erros casuais entre as medidas $\mathrm{F}$ e $\mathrm{T}$ foram muito semelhantes, sendo maiores nas frequências acima de $4 \mathrm{kHz}$. As diferenças e variações entre as medidas $\mathrm{F}$ e T estavam dentro da magnitude de variabilidade do procedimento de medidas com microfone sonda. Conclusão: as medidas com microfone sonda via teleconsulta fornece resultados confiáveis e similares aos obtidos pelo procedimento padrão.
\end{abstract}

DESCRITORES: Audição; Auxiliares de Audição; Perda Auditiva; Telemedicina

\section{INTRODUÇÃO}

A equiparação do desempenho eletroacústico da prótese auditiva com as necessidades de um indivíduo em particular é um fator crítico para o sucesso da adaptação. As simulações da resposta da prótese na orelha do usuário geradas pelos softwares de programação dos fabricantes superestimam a amplificação fornecida, sobretudo em altas frequências ${ }^{1,2}$. Desta forma é determinante

(1) Fonoaudióloga; Professora Doutora do Departamento de Fonoaudiologia da Faculdade de Odontologia de Bauru Universidade de São Paulo, FOB-USP, Bauru, SP, Brasil. Doutora em Neurociências e Comportamento pelo Instituto de Psicologia da Universidade de São Paulo.

(2) Fonoaudióloga do Centro Integrado de Reabilitação - Hospital Estadual de Ribeirão Preto, SP, Brasil; Mestre em Fonoaudiologia pela Faculdade de Odontologia de Bauru - Universidade de São Paulo - FOB -USP.

(3) Fonoaudióloga da Clínica de Fonoaudiologia da Faculdade de Odontologia de Bauru - Universidade de São Paulo, FOB-USP, Bauru, SP, Brasil; Discente do Programa de Mestrado em Fonoaudiologia - FOB/USP.

Conflito de interesses: inexistente que seja realizada a etapa de verificação in situ, isto é, com a prótese no ouvido do usuário, sendo as medidas com microfone sonda o método primário para tal ${ }^{3}$.

Uma característica importante das medidas com microfone sonda é sua confiabilidade, sendo relatada variabilidade teste-reteste menores que 6 $\mathrm{dB}$. As principais fontes de variabilidade são o posicionamento do tubo sonda e quebra da vedação acústica ocasionada pela presença do mesmo no conduto auditivo ${ }^{4}$; a distância e o ângulo de posicionamento do alto falante ${ }^{5}$, o posicionamento do microfone de referência ${ }^{6}$, o método de equalização do campo sonoro ${ }^{7}$, a acústica ambiental e a presença de cerume no conduto auditivo ${ }^{8} \mathrm{e}$ condições da orelha média ${ }^{9}$.

No Brasil existe desigualdade de acesso à educação profissional continuada, gerando diferenças importantes na qualificação profissional de uma região para outra e, por consequência, tornando problemático o acesso a serviços de qualidade. Desta forma novos modelos de serviço devem ser utilizados. A telessaúde consiste no 
fornecimento de serviços de saúde a distância via tecnologias de comunicação e, cada vez mais, vem sendo proposta como uma alternativa ao modelo convencional de fornecimento de serviços presenciais e apresentada como tendo um papel transformador no cuidado à saúde.

A teleconsulta (teleassistência ou teleprática) é definida como a aplicação de tecnologia para enviar serviços de saúde à distância conectando profissional e paciente ou profissional e profissional, fornecendo quaisquer ou todos os seguintes serviços: (a) treinamento, aconselhamento, educação; (b) avaliação para estabelecer a condição de um dado paciente; (c) intervenção e (d) assistência remota para treinamento de profissionais ${ }^{10,11}$.

A teleconsulta pode ser realizada via protocolos assíncronos (armazenamento e envio) ou síncronos. A teleconsulta síncrona é a que mais se aproxima das consultas face a face e permite que profissionais interajam com pacientes ou outros profissionais de saúde utilizando áudio e/ou vídeo em tempo real. Também é possível utilizar softwares de compartilhamento de dados para controlar remotamente aplicativos de um computador e seus periféricos ${ }^{12}$.

No Brasil, o uso da teleconsulta está regulamentado pela Resolução 366 do Conselho Federal de Fonoaudiologia ${ }^{13}$. Dentre outros artigos esta Resolução dispõe que os procedimentos realizados devem garantir a mesma eficácia do atendimento presencial. Deste modo são necessários estudos que comparem a teleconsulta aos procedimentos convenscionais (face a face)

Pesquisadores vêm empregando a teleconsulta síncrona para a realização de diferentes procedimentos audiológicos como a audiometria tonal ${ }^{10}$, emissões otoacústicas evocadas ${ }^{14}$, potenciais auditivos evocados de tronco encefálico ${ }^{15}$ e programação do implante coclear ${ }^{16,17}$. Tais estudos demonstram concordância entre os resultados obtidos com os procedimentos padrão e de teleconsulta.

Pesquisas que avaliaram o uso de teleconsulta, via vídeo interativo e software de compartilhamento de dados, para a programação ${ }^{18} \mathrm{e}$ verificação das próteses auditivas com medidas de microfone sonda ${ }^{19}$ encontraram resultados similares aos obtidos face a face.

Inúmeras próteses auditivas apresentam circuitos de processamento do sinal que modificam a resposta de frequência ou saída frente à análise das características de amplitude, espectro e/ou duração do sinal de entrada. Deste modo é possível que pequenas instabilidades durante a realização da verificação remota causadas, por exemplo, pela perda de informação devido ao excessivo tráfego ou reduzida largura de banda para transmissão de dados, alterem os resultados obtidos. Por esta razão o objetivo deste estudo é analisar as medidas repetidas da resposta de ressonância da orelha externa (REUR), resposta de ressonância da orelha externa com uso da amplificação (REAR) e o ganho de inserção (REIG) realizados face a face e via teleconsulta.

\section{MÉTODO}

Estudo prospectivo longitudinal desenvolvido na Clínica de Fonoaudiologia da Faculdade de Odontologia de Bauru da Universidade de São Paulo (FOB-USP).

Foram avaliados 19 adultos (10 homens 09 mulheres) com idades variando entre 18 a 52 anos (média de 28 anos), que não possuíam queixas auditivas e apresentavam otoscopia e resultados de imitanciometria normais. Os procedimentos foram realizados apenas na orelha direita dos participantes, totalizando 19 orelhas. Todos os participantes foram voluntários tendo assinado o termo de Consentimento Livre e Esclarecido.

Um molde auricular personalizado do tipo invisível simples, em material acrílico, com tubo padrão e sem modificações acústicas foi confeccionado para cada participante.

As medidas com microfone sonda foram realizadas em uma sala acusticamente tratada. O equipamento Unity PC Probe Mic (Siemens) foi utilizado. Antes do posicionamento do paciente no ambiente de teste, foi realizada a calibração do tubo sonda de acordo com as instruções do manual do equipamento. O método de equalização concorrente foi empregado - ou seja, um microfone de referência posicionado na orelha testada monitorava o sinal que atingia o ouvido do participante e realizava, automaticamente, os ajustes dos níveis de pressão sonora (NPS) no alto falante que fossem necessários para atingir o NPS desejado ${ }^{7}$.

A inspeção otológica clínica foi realizada a fim de verificar as condições da orelha externa antes do início das mensurações. O participante foi posicionado no ambiente de teste sentado em uma cadeira localizada a $50 \mathrm{~cm}$ de distância da caixa sonora, posicionada a $0^{\circ}$ azimute. Durante todo o teste foi solicitado ao participante que permanecesse em silêncio, sem movimentar a cabeça, com os braços ao longo do corpo e mãos apoiadas nas coxas.

O método de pressão modificado por equalização concomitante foi utilizado para equalização do ambiente ${ }^{20}$.

Para posicionamento do tubo sonda na orelha do participante foi utilizado o método geométrico. A ponta do tubo sonda foi posicionada a uma distância 
de aproximadamente quatro milímetros da ponta do molde auricular. O tubo sonda foi então cuidadosamente inserido no conduto auditivo externo, permanecendo nesta posição durante toda a avaliação.

As seguintes medidas foram realizadas, na ordem: (1) Resposta de ressonância da orelha externa (REUR); (2) Resposta de ressonância com a prótese auditiva (REAR) e (3) Ganho de inserção (REIG). A REUR e a REAR medem o nível de pressão sonora absoluto, em função da frequência, gerado ao nível da membrana timpânica sem e com a prótese auditiva, respectivamente. O REIG é a medida da diferença entre a REAR e a REUR ${ }^{21}$.

Para todas as medidas foi utilizado o estímulo do tipo speech noise e nível de apresentação igual a 65 dB NPS, sendo este mantido constante.

Para as medidas com uso da amplificação (REAR e REIG) foi utilizada uma prótese auditiva tipo mini-retroauricular cuja média do ganho acústico nas frequencias de $500,1 \mathrm{k}$ e $2 \mathrm{k} \mathrm{Hz}$ era igual a $38 \mathrm{~dB}$ e média da saída, nestas mesmas frequencias, igual a $113 \mathrm{~dB}$ NPS. A prótese não possuía os seguintes recursos: (a) microfone direcional; (b) algoritmo digital de redução de ruído; (c) algoritmo digital de redução ou supressão do feedback e (d) algoritmo digital de destaque espectral. Ressaltase que foram realizados ajustes nas regulagens da prótese de modo a proporcionar amplificação mínima, evitando o desconforto acústico ou possíveis danos à audição dos participantes.

Para a realização do procedimento à distância foi utilizado o protocolo síncrono com o controle remoto de aplicativos (Figura 1). Um facilitador e o participante foram posicionados na sala onde se encontrava o equipamento Unity PC Probe (ambiente de teste) conectado a um computador e à internet (Local Area Network - LAM). A este computador estavam também conectados uma webcam Logitech QuickCam Orbit e um sistema de fone de ouvido e microfone (headset Bright).

Ressalta-se que este facilitador era um fonoaudiólogo, sem experiência prévia no processo de seleção, verificação e adaptação dos aparelhos de amplificação sonora individuais, que se voluntariou para participar da pesquisa.

\section{AMBIENTE REMOTO}

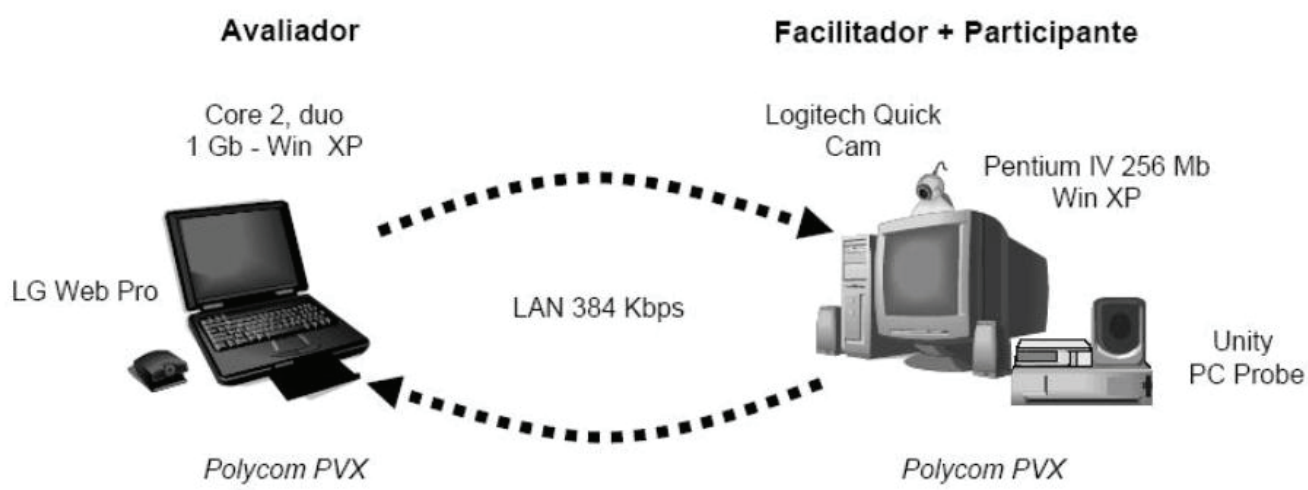

Figura 1 - Representação esquemática do protocolo utilizado para a realização das medidas com microfone sonda via teleconsulta

O avaliador posicionou-se em uma sala (ambiente remoto) localizada a aproximadamente 20 metros de distância do ambiente de teste. Nesta sala havia um notebook conectado a um fone de ouvido e microfone (headset Logitech), à webcam $L G$ WebPro e à $L A N$.

O aplicativo Polycom PVX Version 8.0.2 (Video Voice Data Web) encontrava-se instalado tanto no computador pessoal como no notebook e foi utilizado como software para controle remoto e para transmissão de áudio e vídeo entre os dois ambientes. A taxa de transmissão de $384 \mathrm{Kbps}$ foi utilizada.
O vídeo interativo permitiu que o avaliador fornecesse, em tempo real, as orientações necessárias ao facilitador para posicionamento do participante no ambiente de teste, calibração do tubo sonda e inserção do tubo sonda no conduto auditivo dos participantes via método geométrico.

Uma vez inserido o tubo sonda no conduto auditivo do participante, o avaliador, por intermédio do compartilhamento de dados do software Polycom $P V X$, obtinha o controle do computador e do equipamento Unity $P C$ Probe Mic. Foram então realizadas as medidas da REUR, REAR e REIG. Novamente todas as medidas foram realizadas com 0 
estímulo speech noise e nível de apresentação de $65 \mathrm{~dB}$ NPS.

Durante todo o procedimento das medidas co microfone sonda, uma janela com o registro das respostas era visível em tempo real, tanto para o avaliador como para o facilitador e participante. Assim, com base na análise dessas respostas e na observação do ambiente de teste (via vídeo interativo) era possível detectar erros como a má inserção do tubo sonda ou movimentação do participante. Com isso, as orientações eram repetidas para que o facilitador pudesse recomeçar o procedimento. $\mathrm{O}$ tempo despendido para realização das medidas a distância foi cronometrado.

Foram realizadas quatro repetições das medidas com microfone sonda realizadas face a face e via teleconsulta, ao longo de duas semanas. Para cada comparecimento do participante foram obtidas uma medida face a face e uma medida via teleconsulta, sendo a ordem de realização dos procedimentos contrabalançada.

Este estudo foi analisado e aprovado pelo Comitê de Ética em Pesquisa da Faculdade de Odontologia de Bauru, Universidade de São Paulo - FOB/USP (processo $\mathrm{n}^{\circ}$ 112/2006).

A análise estatística foi realizada com o software Statistica for Windows v 5.1, StatSoft Inc. Foi calculado o erro casual proposto por Dahlberg para verificar o erro obtido nas quatro medidas da REUR, REAR e REIG, nas frequências de 0,25 a $8 \mathrm{kHz}$, realizadas face a face e via teleconsulta. $A$ análise de variância a dois critérios para medidas repetidas (ANOVA) foi utilizada para verificar se existia diferença, considerando as quatro repetições, entre os procedimentos realizados. Em todos os casos foi adotado um nível de significância de 5\%.

\section{RESULTADOS}

Em média foram utilizados 5 minutos e 20 segundos para realização das medidas com microfone sonda via teleconsulta (mínimo de 2 minutos e 34 segundos e máximo de 16 minutos e 51 segundos).

As Tabelas 1 a 3 mostram a média das amplitudes registradas para cada uma das quatro repetições da REUR (Tabela 1), REAR (Tabela 2) e REIG (Tabela 3) obtidas face a face e via teleconsulta, a diferença entre elas e o resultado da análise de variância. Valores negativos de diferença indicam que a amplitude das medidas via teleconsulta foi maior do que a obtida face a face.

A Tabela 4 mostra as médias dos erros obtidos por meio da comparação casual de Dahlberg encontrados nas quatro repetições da REUR, REAR e REIG realizadas face a face e via teleconsulta, em cada frequência pesquisada. A diferença entre os erros dos procedimentos face a face e teleconsulta (face - teleconsulta) também é mostrada. Valores negativos de diferença indicam que o erro para medidas de teleconsulta foi maior que o para as medidas face a face.

\section{DISCUSSÃO}

No que se refere ao tempo médio para realização do procedimento via teleconsulta foram encontrados valores semelhantes ao observado na prática clínica para realização do procedimento da forma padrão (face a face). Ressalta-se, no entanto, que em alguns casos aproximadamente 17 minutos foram necessários para completar a avaliação. O principal fator que influenciou o tempo de execução do procedimento foi a habilidade do facilitador em executar as orientações fornecidas pelo avaliador, sobretudo o posicionamento correto do tubo sonda no conduto auditivo externo do participante. Quando observadas alterações nas respostas registradas em função do mau posicionamento do tubo sonda e/ou molde auricular no conduto auditivo, compressão do tubo sonda pelo molde auricular ou, ainda, presença de cerume obstruindo o tubo sonda, interrupções no exame eram realizadas pelo avaliador a fim de orientar o facilitador para sanar tais problemas e recomeçar a avaliação.

Quanto às medidas da REUR (Tabela 1), REAR (Tabela 2) e REIG (Tabela 3), considerando todas as repetições, as diferenças entre as medidas face a face e teleconsulta variaram entre 0 a $1,7 \mathrm{~dB}$ (REUR) e 0 a 2,3 dB (REAR e REIG), não sendo significantes. Ferrari e Bernardez-Braga ${ }^{19}$ também não encontraram diferenças quando comparado os procedimentos face a face e de teleconsulta para as medidas de REUR, REAG e REIG obtidas em adultos e idosos com deficiência auditiva.

Deve ser ressaltada que as amplitudes baixas da REAR e REIG e as amplitudes negativas do REIG encontradas neste estudo ocorreram pelo fato da prótese utilizada ter sido ajustada propositalmente para propiciar a menor amplificação possível.

Foi calculada a média dos erros casuais ou aleatórios entre as quatro medições realizadas (Tabela 4). Considerando a REUR, o erro para medidas face a face variou de $2,12 \mathrm{~dB}(500 \mathrm{~Hz})$ a $5,57 \mathrm{~dB}(8 \mathrm{kHz})$ e via teleconsulta variou de 2,36 $\mathrm{dB}(1 \mathrm{kHz})$ a $6,55 \mathrm{~dB}(8 \mathrm{kHz})$. Os erros obtidos para as medidas face a face foram menores do que os obtidos via teleconsulta em todas as frequências analisadas, no entanto, a diferença entre os mesmos não foi superior a $0,98 \mathrm{~dB}$. Erros maiores foram observados para as frequências acima de $4 \mathrm{kHz}$. Esses dados corroboram os resultados de 
Tabela 1 - Média e desvio padrão das amplitudes (dB NPS) registradas para as quatro medidas da resposta de ressonância da orelha externa (REUR), realizadas face a face e via teleconsulta e a diferença entre estas ( $N=19)$

\begin{tabular}{|c|c|c|c|c|c|c|c|}
\hline \multirow{2}{*}{ Freq $(\mathrm{Hz})$} & \multirow{2}{*}{ Repetição } & \multicolumn{2}{|c|}{ Face a face } & \multicolumn{2}{|c|}{ Teleconsulta } & \multirow{2}{*}{ Diferença } & \multirow{2}{*}{ Anova } \\
\hline & & $x$ & $\mathrm{dp}$ & $x$ & $d p$ & & \\
\hline \multirow{4}{*}{250} & 1 & 61,2 & 4,1 & 60,1 & 5,8 & 1,1 & \multirow{4}{*}{0,67} \\
\hline & 2 & 63,3 & 0,7 & 63,3 & 1,4 & 0,0 & \\
\hline & 3 & 61,7 & 4,3 & 62,0 & 3,9 & $-0,3$ & \\
\hline & 4 & 63,5 & 0,9 & 63,7 & 1,2 & $-0,2$ & \\
\hline \multirow{4}{*}{500} & 1 & 63,2 & 3,7 & 62,4 & 4,1 & 0,8 & \multirow{4}{*}{0,53} \\
\hline & 2 & 64,7 & 0,8 & 64,8 & 0,6 & $-0,1$ & \\
\hline & 3 & 64,1 & 2,8 & 64,0 & 3,2 & 0,1 & \\
\hline & 4 & 64,8 & 0,5 & 64,7 & 0,8 & 0,1 & \\
\hline \multirow{4}{*}{1000} & 1 & 65,7 & 3,7 & 65,0 & 3,3 & 0,7 & \multirow{4}{*}{0,65} \\
\hline & 2 & 66,5 & 1,1 & 67,0 & 1,5 & 0,5 & \\
\hline & 3 & 65,5 & 3,2 & 66,0 & 2,9 & 0,5 & \\
\hline & 4 & 66,8 & 1,0 & 67,0 & 1,2 & $-0,2$ & \\
\hline \multirow{4}{*}{2000} & 1 & 73,0 & 4,4 & 71,7 & 5,0 & 1,3 & \multirow{4}{*}{0,30} \\
\hline & 2 & 75,6 & 1,9 & 75,2 & 2,4 & 0,4 & \\
\hline & 3 & 74,0 & 3,9 & 74,0 & 4,5 & 0,0 & \\
\hline & 4 & 75,2 & 1,4 & 74,9 & 2,1 & 0,3 & \\
\hline \multirow{4}{*}{3000} & 1 & 77,2 & 5,6 & 75,9 & 6,4 & 1,3 & \multirow{4}{*}{0,36} \\
\hline & 2 & 79,3 & 2,1 & 79,2 & 3,0 & 0,1 & \\
\hline & 3 & 78,5 & 5,3 & 78,1 & 4,4 & 0,4 & \\
\hline & 4 & 79,4 & 2,0 & 79,4 & 2,4 & 0,0 & \\
\hline \multirow{4}{*}{4000} & 1 & 74,7 & 6,8 & 72,6 & 7,1 & 2,1 & \multirow{4}{*}{0,24} \\
\hline & 2 & 76,8 & 2,5 & 75,7 & 2,9 & 1,1 & \\
\hline & 3 & 75,4 & 5,7 & 75,8 & 4,3 & $-0,4$ & \\
\hline & 4 & 76,1 & 2,9 & 76,0 & 2,5 & 0,1 & \\
\hline \multirow{4}{*}{6000} & 1 & 64,1 & 10,0 & 63,1 & 8,1 & 1,0 & \multirow{4}{*}{0,91} \\
\hline & 2 & 65,6 & 5,6 & 65,1 & 5,0 & 0,5 & \\
\hline & 3 & 65,1 & 8,6 & 66,0 & 7,7 & $-0,9$ & \\
\hline & 4 & 66,2 & 3,7 & 67,1 & 5,5 & $-0,9$ & \\
\hline \multirow{4}{*}{8000} & 1 & 58,2 & 10 & 59,1 & 9,1 & $-0,9$ & \multirow{4}{*}{0,63} \\
\hline & 2 & 61,2 & 5,8 & 59,5 & 7,1 & 1,7 & \\
\hline & 3 & 58,6 & 8,2 & 60,1 & 6,3 & $-1,5$ & \\
\hline & 4 & 59,9 & 4,6 & 60,7 & 5,5 & $-0,8$ & \\
\hline
\end{tabular}

${ }^{*} p<0,05$ estatisticamente significante

outras pesquisas onde foi verificado o aumento da variabilidade das medidas da REUR com o aumento da frequência ${ }^{22}$ e diferenças entre as medidas da REUR variando de $1(250 \mathrm{~Hz})$ a $3,41 \mathrm{~dB}(4 \mathrm{kHz})^{23}$.

Para a REAR o erro para medidas face a face variou de $3,01 \mathrm{~dB}(250 \mathrm{~Hz})$ a $6,75 \mathrm{~dB}(8 \mathrm{kHz})$ e à distância variou de 2,88 dB $(500 \mathrm{~Hz})$ a $5,74 \mathrm{~dB}(8$ $\mathrm{kHz}$ ). O erro para as medidas face a face foi, em média, apenas $0,1 \mathrm{~dB}$ menor do que o das medidas à distância. Resultados semelhantes foram descritos para as medidas da REAR, com erros de aproximadamente 2-3 dB para as frequências abaixo de $1 \mathrm{kHz}, 3 \mathrm{~dB}$ na região de 1 a $2 \mathrm{kHz}$ e $4 \mathrm{~dB}$ nas frequências iguais ou acima de $3 \mathrm{kHz}{ }^{24}$.

Finalmente, para o REIG o erro para medidas face a face variou de $1,40 \mathrm{~dB}(250$ e $500 \mathrm{~Hz})$ a 5,04 $\mathrm{dB}(8 \mathrm{kHz})$ e via teleconsulta variou de $1,40 \mathrm{~dB}$ (250 $\mathrm{Hz})$ a $5,38 \mathrm{~dB}(8 \mathrm{kHz})$. O erro para as medidas face a face foi em média apenas $0,08 \mathrm{~dB}$ menor do que 0 das medidas à distância. Os erros verificados para 
Tabela 2 - Média e desvio padrão das amplitudes (dB NPS) registradas para as quatro medidas da resposta de ressonância da orelha externa com uso da amplificação (REAR), realizadas face a face e via teleconsulta e a diferença entre estas $(N=19)$

\begin{tabular}{|c|c|c|c|c|c|c|c|}
\hline \multirow{2}{*}{ Freq (Hz) } & \multirow{2}{*}{ Repetição } & \multicolumn{2}{|c|}{ Face a face } & \multicolumn{2}{|c|}{ Teleconsulta } & \multirow{2}{*}{ Diferença } & \multirow{2}{*}{ Anova } \\
\hline & & $x$ & $d p$ & $x$ & $d p$ & & \\
\hline \multirow{4}{*}{250} & 1 & 61,3 & 5,1 & 60,5 & 5,6 & 0,8 & \multirow{4}{*}{1,0} \\
\hline & 2 & 63,8 & 0,8 & 63,5 & 1,4 & 0,3 & \\
\hline & 3 & 61,7 & 4,0 & 62,7 & 3,4 & $-1,0$ & \\
\hline & 4 & 63,7 & 1,5 & 63,7 & 1,3 & 0,0 & \\
\hline \multirow{4}{*}{500} & 1 & 63,9 & 4,1 & 64,0 & 4,5 & $-0,1$ & \multirow{4}{*}{0,61} \\
\hline & 2 & 66,8 & 1,6 & 66,0 & 1,7 & 0,8 & \\
\hline & 3 & 64,8 & 4,1 & 64,8 & 3,2 & 0,0 & \\
\hline & 4 & 66,1 & 2,5 & 65,8 & 2,4 & 0,3 & \\
\hline \multirow{4}{*}{1000} & 1 & 73,7 & 3,75 & 74,4 & 3,8 & $-0,7$ & \multirow{4}{*}{0,83} \\
\hline & 2 & 74,2 & 3,8 & 74,6 & 3,7 & $-0,4$ & \\
\hline & 3 & 73,2 & 4,5 & 72,7 & 4,4 & 0,5 & \\
\hline & 4 & 73,9 & 3,5 & 73,8 & 4,0 & 0,1 & \\
\hline \multirow{4}{*}{2000} & 1 & 84,9 & 4,8 & 83,5 & 5,9 & 0,9 & \multirow{4}{*}{0,06} \\
\hline & 2 & 85,5 & 2,9 & 84,9 & 3,9 & 0,6 & \\
\hline & 3 & 85,1 & 5,4 & 83,8 & 4,7 & 1,3 & \\
\hline & 4 & 84,5 & 3,0 & 84,5 & 5,3 & 0,0 & \\
\hline \multirow{4}{*}{3000} & 1 & 85,8 & 6,3 & 84,8 & 5,9 & 1,0 & \multirow{4}{*}{0,65} \\
\hline & 2 & 86,2 & 3,4 & 86,7 & 3,9 & $-0,5$ & \\
\hline & 3 & 86,2 & 6,0 & 85,7 & 4,8 & 0,5 & \\
\hline & 4 & 86,8 & 3,5 & 86,9 & 4,9 & $-0,1$ & \\
\hline \multirow{4}{*}{4000} & 1 & 77,9 & 6,0 & 76,4 & 6,3 & 1,5 & \multirow{4}{*}{0,20} \\
\hline & 2 & 77,4 & 3,9 & 77,3 & 4,1 & 0,1 & \\
\hline & 3 & 77,9 & 5,4 & 76,0 & 4,8 & 1,9 & \\
\hline & 4 & 76,5 & 4,7 & 77,4 & 5,7 & $-0,9$ & \\
\hline \multirow{4}{*}{6000} & 1 & 60,3 & 8,4 & 58,8 & 7,4 & 1,5 & \multirow{4}{*}{0,12} \\
\hline & 2 & 62,4 & 4,4 & 61,9 & 5,6 & 0,5 & \\
\hline & 3 & 63,3 & 7,1 & 61,2 & 8,2 & 2,1 & \\
\hline & 4 & 61,9 & 5,1 & 62,1 & 6,1 & $-0,2$ & \\
\hline \multirow{4}{*}{8000} & 1 & 53,7 & 7,6 & 53,8 & 8,5 & $-0,1$ & \multirow{4}{*}{0,86} \\
\hline & 2 & 57,7 & 5,8 & 57,2 & 4,9 & 0,5 & \\
\hline & 3 & 57,8 & 7,8 & 56,9 & 7,8 & 0,9 & \\
\hline & 4 & 56,6 & 6,6 & 57,4 & 4,0 & $-0,8$ & \\
\hline
\end{tabular}

${ }^{*} \mathrm{p}<0,05$ estatisticamente significante

a medida do ganho de inserção provavelmente estão relacionados a um pequeno deslocamento do tubo sonda para a realização da medida da REAR em relação à posição inicial utilizada para a medida da REUR. Quando isto ocorre observamse variações nos registros do REIG para frequências acima de $2000 \mathrm{~Hz}$ sendo estas menores do que $4 \mathrm{~dB}^{25}$.

Os resultados do presente estudo estão em concordância com a literatura que relata diferenças entre duas repetições da REIG variando de 1,6 $(250 \mathrm{~Hz})$ a $2,1 \mathrm{~dB}(3 \mathrm{kHz}){ }^{26}$. Hawkins ${ }^{25}$ também encontrou erros na REIG em torno de 2,2 dB em 1 $\mathrm{kHz}$. Para a frequência de $6 \mathrm{kHz}$ o autor encontrou erros ainda maiores do que o do presente estudo, na ordem de $7 \mathrm{~dB}$.

No tocante às medidas realizadas à distância, os dados da Tabela 4 corroboram o estudo de Ferrari ${ }^{18}$ que verificou que a média do erro casual para três repetições do ganho de inserção realizado 
Tabela 3 - Média e desvio padrão da amplitude (dB) registrado para as quatro medidas do ganho de inserção (REIG), realizadas face a face e via teleconsulta e a diferença entre estas $(N=19)$

\begin{tabular}{|c|c|c|c|c|c|c|c|}
\hline \multirow{2}{*}{ Freq (Hz) } & \multirow{2}{*}{ Repetição } & \multicolumn{2}{|c|}{ Face a face } & \multicolumn{2}{|c|}{ Teleconsulta } & \multirow{2}{*}{ Diferença } & \multirow{2}{*}{ Anove } \\
\hline & & $x$ & $\mathrm{dp}$ & $\mathbf{x}$ & $d p$ & & \\
\hline \multirow{4}{*}{250} & 1 & $-0,4$ & 1,7 & $-0,3$ & 2,1 & 0,1 & \multirow{4}{*}{0,71} \\
\hline & 2 & 0,8 & 1,4 & 0,3 & 1,1 & 0,5 & \\
\hline & 3 & 0,2 & 1,6 & 0,5 & 1,5 & $-0,3$ & \\
\hline & 4 & $-0,1$ & 1,8 & $-0,3$ & 1,8 & $-0,2$ & \\
\hline \multirow{4}{*}{500} & 1 & 0,4 & 2,3 & 1,1 & 1,9 & $-0,7$ & \multirow{4}{*}{0,61} \\
\hline & 2 & 1,6 & 1,3 & 0,9 & 1,7 & 0,7 & \\
\hline & 3 & 0,8 & 2,1 & 0,4 & 1,9 & 0,4 & \\
\hline & 4 & 0,8 & 1,9 & 0,7 & 2,7 & 0,1 & \\
\hline \multirow{4}{*}{1000} & 1 & 7,9 & 1,9 & 8,6 & 2,1 & $-0,7$ & \multirow{4}{*}{0,83} \\
\hline & 2 & 7,1 & 3,4 & 6,5 & 3,3 & 0,6 & \\
\hline & 3 & 6,6 & 2,7 & 6,2 & 3,0 & 0,4 & \\
\hline & 4 & 6,6 & 2,6 & 7,2 & 2,6 & $-0,6$ & \\
\hline \multirow{4}{*}{2000} & 1 & 11,0 & 3,7 & 11,0 & 4,3 & 0,0 & \multirow{4}{*}{0,21} \\
\hline & 2 & 9,4 & 3,1 & 9,2 & 4,1 & 0,2 & \\
\hline & 3 & 9,9 & 4,0 & 9,7 & 3,3 & 0,2 & \\
\hline & 4 & 8,4 & 4,1 & 7,2 & 2,6 & 1,2 & \\
\hline \multirow{4}{*}{3000} & 1 & 7,8 & 4,6 & 8,0 & 4,9 & $-0,2$ & \multirow{4}{*}{0,27} \\
\hline & 2 & 6,5 & 3,3 & 6,7 & 4,5 & $-0,2$ & \\
\hline & 3 & 6,7 & 3,5 & 7,2 & 3,6 & $-0,5$ & \\
\hline & 4 & 6,6 & 4,3 & 7,5 & 4,8 & $-0,9$ & \\
\hline \multirow{4}{*}{4000} & 1 & 1,8 & 4,1 & 2,5 & 4,4 & $-0,7$ & \multirow{4}{*}{0,23} \\
\hline & 2 & 0,4 & 2,7 & 1,4 & 4,1 & $-1,0$ & \\
\hline & 3 & 1,3 & 2,8 & 0,2 & 3,2 & 1,1 & \\
\hline & 4 & $-0,4$ & 4,5 & 1,5 & 5,0 & $-0,9$ & \\
\hline \multirow{4}{*}{6000} & 1 & $-4,1$ & 5,0 & $-4,2$ & 6,9 & $-0,1$ & \multirow{4}{*}{0,37} \\
\hline & 2 & $-2,9$ & 5,1 & $-3,3$ & 4,9 & $-0,4$ & \\
\hline & 3 & $-2,6$ & 4,9 & $-4,9$ & 4,6 & $-2,3$ & \\
\hline & 4 & $-4,7$ & 4,6 & $-4,1$ & 4,5 & 0,6 & \\
\hline \multirow{4}{*}{8000} & 1 & $-3,6$ & 6,7 & $-5,5$ & 7,6 & $-1,9$ & \multirow{4}{*}{0,37} \\
\hline & 2 & $-3,0$ & 6,1 & $-1,5$ & 6,8 & 1,5 & \\
\hline & 3 & $-2,6$ & 7,9 & $-3,7$ & 4,5 & $-1,1$ & \\
\hline & 4 & $-1,6$ & 5,1 & $-3,6$ & 5,2 & $-2,0$ & \\
\hline
\end{tabular}

${ }^{*} \mathrm{p}<0,05$ estatisticamente significante

via controle remoto do equipamento variou entre $0,7(250 \mathrm{~Hz})$ a $1,44 \mathrm{~dB}(4 \mathrm{kHz})$.

Observa-se que tanto para as medidas face a face como para a teleconsulta os erros foram maiores nas frequências acima de $4 \mathrm{kHz}$. Tal resultado pode ter ocorrido em virtude das altas frequências sofrerem maior influência da profundidade de inserção do tubo sonda entre as diferentes medições. A maior susceptibilidade das altas frequências pode ser explicada pelo fenômeno das ondas estacionárias. A onda estacionária acontece quando da somação da onda sonora incidente com parte da onda sonora refletida pela membrana timpânica. Quando a distância percorrida pela onda sonora refletida for igual a $1 / 4$ do comprimento da onda sonora incidente, ocorre o cancelamento parcial da onda, resultando numa atenuação importante do NPS gerado no conduto auditivo externo.

O comprimento de onda das frequências abaixo de $1 \mathrm{kHz}$ é longo em relação ao comprimento médio do conduto auditivo externo de adultos (em média $25 \mathrm{~mm}$ ) e, portanto, não sofrem interferência das 
Tabela 4 - Médias dos erros (Dahlberg) encontrado nas quatro repetições da REUR, REAR e REIG realizadas face a face e via teleconsulta, e diferença entre eles $(\mathrm{N}=19)$

\begin{tabular}{cccccccccc}
\hline & \multicolumn{3}{c}{ REUR } & \multicolumn{3}{c}{ REAR } & \multicolumn{3}{c}{ REIG } \\
\cline { 2 - 10 } & Face & Tele & Dif & Face & Tele & Dif & Face & Tele & Dif \\
\hline $\mathbf{2 5 0}$ & 2,92 & 3,73 & $-0,81$ & 3,01 & 3,38 & $-0,37$ & 1,40 & 1,40 & 0 \\
$\mathbf{5 0 0}$ & 2,12 & 2,63 & $-0,51$ & 3,02 & 2,88 & 0,14 & 1,40 & 1,63 & $-0,23$ \\
$\mathbf{1 0 0 0}$ & 2,35 & 2,36 & $-0,01$ & 3,16 & 3,52 & $-0,36$ & 2,45 & 2,42 & 0,03 \\
$\mathbf{2 0 0 0}$ & 3,13 & 3,51 & $-0,38$ & 3,24 & 4,00 & $-0,76$ & 2,48 & 2,10 & 0,38 \\
$\mathbf{3 0 0 0}$ & 3,15 & 3,94 & $-0,79$ & 3,23 & 3,79 & $-0,56$ & 2,23 & 2,33 & $-0,1$ \\
$\mathbf{4 0 0 0}$ & 3,71 & 4,01 & $-0,3$ & 3,60 & 3,55 & 0,05 & 2,72 & 2,79 & $-0,07$ \\
$\mathbf{6 0 0 0}$ & 5,03 & 5,58 & $-0,55$ & 5,17 & 5,27 & $-0,1$ & 4,09 & 4,46 & $-0,37$ \\
$\mathbf{8 0 0 0}$ & 5,57 & 6,55 & $-0,98$ & 6,75 & 5,74 & 1,01 & 5,04 & 5,38 & $-0,34$ \\
\hline
\end{tabular}

Legenda: Face: face a face; Tele: Teleconsulta; Dif: Diferença.

ondas estacionárias geradas no mesmo. Em consequência, no caso das frequências baixas o tubo sonda posicionado em qualquer lugar do conduto irá medir, essencialmente, um nível de pressão sonora igual ao gerado próximo na membrana timpânica ${ }^{4}$. As altas frequências, em virtude do seu comprimento de onda, sofrem o efeito das ondas estacionárias e, assim, maior variabilidade será encontrada na dependência da posição do tubo sonda. As variações encontradas nas frequências acima de $3000 \mathrm{~Hz}$ variam de $2 \mathrm{a} 10 \mathrm{~dB}^{27}$.

No que se refere à medida da REAR e REIG outro fator que também afeta a medida em alta frequência e, por conseguinte, sua variabilidade, é a existência da turbulência que ocorre na região da abertura do molde auricular ${ }^{8}$. Quando um som irradia de um tubo pequeno (abertura do molde) para um ducto maior (conduto auditivo) ondas radiais e longitudinais se desenvolvem nessa área de transição. A interação entre essas ondas pode causar variação de 5 a $12 \mathrm{~dB}$ no NPS nas frequências entre $2 \mathrm{k}$ e $8 \mathrm{kHz}$ caso o tubo sonda fique próximo a esta zona de turbulência ${ }^{4,27}$. No presente estudo o molde auricular foi utilizado como guia para o posicionamento da ponta do tubo sonda após esta área de transição, no entanto, durante a própria inserção do molde auricular na orelha externa do participante pode ter ocorrido deslocamento do tubo para esta região de turbulência.

Movimentações de cabeça podem resultar em inconsistências no sinal que incide na orelha testada. Estes efeitos podem ser minimizados quando o altofalante é posicionado em 0 azimute e até $50 \mathrm{~cm}$ de distância do indivíduo ${ }^{5}$, como foi o caso do presente estudo. Movimentações da cabeça no plano horizontal, em relação à posição da fonte sonora, durante a realização do teste podem alterar o nível de pressão sonora fornecido pelo AASI no conduto auditivo em até $7 \mathrm{~dB}{ }^{24}$. Tais movimentos afetam principalmente as altas frequências.

No tocante à REAR e REIG outra hipótese diz respeito ao ajuste do controle de volume manual da prótese auditiva utilizada. O volume da prótese foi mantido na posição "1" que fornecia a menor quantidade de amplificação. No entanto, por este controle ser manual pode não ter ocorrido a replicação exata do posicionamento do mesmo nas diferentes repetições. Alterações da posição do controle de volume acarretam modificações no ganho fornecido pela prótese e, por conseguinte, na amplitude das medidas da REAR e do REIG.

Deve também ser ressaltado que neste estudo cinco voluntários participaram como facilitadores para a realização das medidas no ambiente de teste. Assim, os erros aqui apresentados podem também estar relacionados às variações das habilidades dos facilitadores em executar os procedimentos solicitados pelo especialista.

De qualquer forma, como os erros casuais das medidas realizadas via teleconsulta são muitos similares aos obtidos face a face, é possível concluir que não houve influência importante da realização das medidas com microfone sonda por meio do controle remoto do equipamento. A viabilidade $e$ confiabilidade da teleconsulta para realização de consultas e procedimentos audiológicos à distância foi verificada também em outros estudos ${ }^{14,16,18,28,29}$.

Uma das preocupações com relação à teleconsulta é a consistência e qualidade do envio das informações de um local ao outro. Estudos ${ }^{15,30,31}$ relatam falhas no sinal de áudio e na transmissão dos dados do teste realizado à distância, em momentos de pico na rede, quando o tráfego na internet era intenso, fazendo com que a informação enviada pelo computador remoto não chegasse de maneira ótima no ambiente de 
teste. No caso de exames audiológicos realizados via compartilhamento remoto de aplicativos, estas falhas de transmissão podem levar a registros ou interpretações errôneas de resultados. Desta forma cuidado deve ser tomado quando da utilização de redes como a internet já que o compartilhamento da largura de banda com outros tipos de dados pode fazer com que haja uma perda significante da qualidade dos sinais de áudio e vídeo transmitidos.

A largura de banda é o requisito fundamental para que haja espaço suficiente para que todos os pacotes de informação sejam transmitidos. É comum na videoconferência por IP (Internet Protocol) a utilização de largura de banda por volta de 384 a $768 \mathrm{Kbps}$. No presente estudo a taxa de transmissão utilizada foi de $384 \mathrm{Kbps}$. É importante ainda que os pontos envolvidos na videoconferência sejam capazes de enviar e receber a mesma quantidade de dados para a chamada, ou seja, que exista simetria entre o ambiente remoto e de teste. Quando a largura de banda não é suficiente pode ocorrer a perda de pacotes da informação, o que resulta na interrupção do sinal de áudio bem como em alterações na imagem (por exemplo, congelamento, imagem em "blocos" ou áreas em branco dentro da janela de vídeo).

Como as dados relativos a áudio e vídeo são sensíveis ao tempo, a sincronia também é de grande importância. O processo de codificação e decodificação da informação (dependente tanto do equipamento utilizado como do tempo de tráfego dos pacotes na rede) pode introduzir um atraso ou latência na videoconferência. Se esse atraso for excessivo o processo de comunicação fica bastante prejudicado tanto pela falta de sincronia entre o movimento de lábios com o sinal de áudio como também pelo fato de uma pessoa "falar sobre a outra".

Neste estudo a largura de banda da $L A N$ foi suficiente para evitar que estes fenômenos ocorressem. Assim, durante a coleta não houve queda da conexão utilizada, atrasos na transferência dos dados, bem como falhas na transmissão de áudio e vídeo. O software utilizado permitiu ótima qualidade de imagem entre os dois ambientes (remoto e de teste). Por meio desse software e das câmeras foi possível visualizar o participante e o facilitador sem dificuldades e assim, orientar o profissional em suas tarefas para permitir a realização das medidas necessárias. No entanto, não é possível garantir o mesmo desempenho em redes com largura de banda menores.

\section{CONCLUSÃO}

A realização de medidas com microfone sonda via teleconsulta, em adultos deficientes auditivos, fornece resultados confiáveis e com variabilidade similar à obtida com o procedimento padrão.

\section{ABSTRACT}

Purpose: to evaluate the repeated measurements of the real ear unaided response (REUR), real ear aided response (REAR) and insertion gain (REIG) conducted as face to face (F) and via telecounselling $(T)$. Method: longitudinal prospective study. Four measures of REUR, REAR and REIG (carried out with speech noise stimulus presented at $65 \mathrm{~dB}$ SPL at $0^{\circ}$ azimuth) were obtained in 19 ears in normal hearing adults, face to face and via synchronous telecounsellings with remote control of the real ear equipment and interactive video. Polycom PVX software was used for desktop sharing and video and audio transmission. The Loca Area Network (LAN) USP was used for the connection (transmission rate: $384 \mathrm{kbps}$ ). Dahlberg's casual errors were calculated for obtaining the measures for frequencies from 250 to $8000 \mathrm{~Hz}$. Results: casual errors between $\mathrm{F}$ and $\mathrm{T}$ measures were very similar, being greater for frequencies above $4 \mathrm{kHz}$. The differences between $\mathrm{F}$ and $\mathrm{T}$ measures were no greater than the variability reported for real ear measures. Conclusion: probe microphone measures obtained via telecounselling provides reliable results and similar to those obtained by standard procedures.

KEYWORDS: Hearing; Hearing Aids; Hearing Loss; Telemedicine 


\section{REFERÊNCIAS}

1. Hawkins DB, Cook JA. Hearing aid software predictive gain values: how accurate are they? Hear J. 2003;56(7):26-34.

2. Aarts NL, Caffee CS. Manufacturer predicted and measured REAR values in adult hearing aid fitting: accuracy and clinical usefulness. Int J Audiol. 2005; 44(5):293-301.

3. American Academy of Audiology. Guidelines for the audiologic management of adult hearing impairment. United States: AAA, 2008.

4. Dirks DD, Ahlstrom JB, Eisenberg LS. Effects of probe insertion depth on real ear measurements. Otolaryngol Head Neck Surg. 1994; 110(1):64-74.

5. Stone MA, Moore BCJ. Estimated variability of real-ear insertion response (REIR) due to loudspeaker type and placement. Int $\mathrm{J}$ Audiol. 2004;43(5):271-5.

6. Ickes MA, Hawkins DB, Cooper WA. Effect of reference microphone location and loudspeaker azimuth on probe tube microphone measurements. J Am Acad Audiol. 1991 Jul;2(3):156-63.

7. Lantz J, Jensen OD, Haastrup A, Olsen SO. Real-ear measurement verification for open, non-occluding hearing instruments. Int $\mathrm{J}$ Audiol. 2007;46(1):11-6.

8. Dillon $\mathrm{H}$. Electroacustic Performance and Measurement. In: Dillon H. Hear Aids. Nova York: Thieme; 2001. p. 74-113.

9. Bernardez GRA, Assumpção MT, Coube CZV, Ferrari DV. Efeito da otite média crônica sobre a ressonância da orelha externa. In: Anais do 20응 Encontro Internacional de Audiologia; 2005, São Paulo, SP. São Paulo: Academia Brasileira de Audiologia; 2005.

10. Givens DG, Elangovan S. Internet application to tele-audiology - "nothing' but net". Am J Audiol. 2003;12(2):56-65.

11. American Speech-Language-Hearing Association. Audiologists providing clinical services via telepractice: technical report. United States: ASHA, 2005.

12. Krumm M, Ferrari DV. Contemporary telehealth and telemedicine applications in audiology. Audiology Today. 2008;20:38-41.

13. Conselho Federal de Fonoaudiologia. Resolução no. 366 de 25 de abril de 2009. Dispõe sobre a regulamentação do uso do sistema Telessaúde em Fonoaudiologia. Conselho Federal de Fonoaudilogia, Brasília DF, 25 de abril de 2009.

14. Krumm M, Ribera J, Klich R. Providing basic hearing tests using remote computing technology. J Telemed Telecare. 2007;13(8):406-10.
15. Towers AD, Pisa J, Froelich TM, Krumm M. The reliability of click evoked and frequency specific auditory brainstem response testing using telehealth technology. Semin Hear. 2005;26(1):26-34.

16. Ramos A, Rodrigues C, Martinez-Beneyto P, Perez D, Gault A, Falcon JC. et al. Use of telemedicine in the remote programming of cochlear implants. Acta Otolaryngol. 2009;129(5):533-40.

17. Zumpano CE, Bevilacqua MC, Frederigue-Lopes NB, Costa OA. Programação remota dos sistemas de implante coclear. Rev Soc Bras Fonoaudiol. Brasil. 2009;14(4):539-46.

18. Ferrari DV. Remote programming and verification as a mean to improve quality of hearing aid fitting. In: Rasmussen AN. et al. (Org.). Hearing aid fitting. Centertryk: Danavox, 2006;1:531-44.

19. Ferrari DV, Bernardez-Braga GRA. Remote verification of hearing aid performance. J Telemed Telecare. 2009;15(3):122-4.

20. Shaw P. Are real-ear measurements (REM) accurate when using the modified pressure with stored equalization (MPSE) method? Int $\mathrm{J}$ Audiol. 2010 Jun;49(6):463-6.

21. American National Standards Institute. (1997). Methods of Measurement of Real-Ear Performance Characteristics of Hearing Aids. ANSI S3.46-1997. New York: American National Standards Institute Inc.

22. Barlow NL, Auslander MC, Rines $D$, Stelmachowicz PG. Probe-tube microphone measures in hearing-impaired children and adults. Ear Hear. 1988;9(5):243-7.

23. Valente M, Valente M, Goebel J. Reliability and intersubject variability of the real ear unaided response. Ear Hear. 1991;12(3):216-20.

24. Hawkins DB, Mueller HG. Procedural considerations in probe-microphone measuements. In: Mueller HG, Hawkins DB, Northern JL. Probe microphone measurements: hearing aid selection and assessment. San Diego: Singular, 1992; p. 67-90.

25. Hawkins DB. Clinical ear canal probe tube measures. Ear Hear. 1987;8 Suppl 5:74-81.

26. Valente M, Potts LG, Valente M, Vass W, Goebel J. Intersubject variability of real ear sound pressure level: conventional and insert earphones. J Am Acad Audiol. 1994;5(6):390-8.

27. Dirks DD, Kincaid GE. Basic acoustic considerations of ear canal probe measurements. Ear Hear. 1987;8 Suppl 5:60-7.

28. Krumm, M, Huffman $T$, Dick $K$, Klich $R$. Telemedicine for audiology screening of infants. J Telemed Telecare. 2008;14(2):102-4. 
29. Shapiro H, Huang $T$, Shaw $T$, Roland JT, Lalwani AK. Remote intraoperative monitoring during cochlear implant surgery is feasible and efficient. Otol Neurotol. 2008;29(4):495-8.

30. Ribera JE. Interjudge reliability and validation of telehealth applications of the Hearing in Noise Test. Semin Hear. 2005;26(1):13-8.
31. Krumm M, Ribera J, Schmiedge J. Using a telehealth medium for objective hearing testing: implications for supporting rural universal newborn hearing screening programs. Semin Hear. 2005;26(1):3-12.

http://dx.doi.org/10.1590/S1516-18462011005000104

RECEBIDO EM: 22/03/2011

ACEITO EM: 03/05/2011

Endereço para correspondência

Deborah Viviane Ferrari

Faculdade de Odontologia de Bauru - USP

Departamento de Fonoaudiologia

Al. Dr. Octávio Pinheiro Brisolla 9-75

Bauru - SP

CEP: 17102-101

E-mail: deborahferrari@usp.br 